\title{
A Sheet Structured MOF Magnet: Poly[(1,10-phenanthroline)tetrakis(imidazolato)diiron(II)]
}

Tim Storr, ${ }^{\mathrm{a}, \mathrm{b}, *}$ John R. Thompson, ${ }^{\mathrm{b}}$ Brian O. Patrick, ${ }^{\mathrm{a}}$ William M. Reiff, ${ }^{\mathrm{c}, *}$ Alan Storr, ${ }^{\mathrm{a}, *}$ and Robert C. Thompson ${ }^{\mathrm{a}, *}$

${ }^{a}$ Department of Chemistry, University of British Columbia, Vancouver, B.C., V6T 1Z1, Canada

${ }^{\mathrm{b}}$ Department of Chemistry, Simon Fraser University, Burnaby, B.C., V5A 1S6, Canada

${ }^{c}$ Department of Chemistry, Northeastern University, Boston, MA 02115, USA

Correspondence to: tim_storr@sfu.ca, w.reiff@neu.edu, storr@.chem.ubc.ca and thompson@chem.ubc.ca 


\begin{abstract}
We report the synthesis and structural and magnetic characterization of Poly[(1,10phenanthroline)tetrakis(imidazolato)diiron(II)], $\left[\mathrm{Fe}_{2}(\text { imid })_{4}(\text { phen })\right]_{\mathrm{x}}, \quad \mathbf{1}$. The extended structure of $\mathbf{1}$ is that of a metal organic framework (MOF) involving double layer sheets (bilayers) of alternating tetrahedral and octahedral irons singly bridged by imidazolate ligands. The octahedral iron centers are each additionally coordinated by phenanthroline ligands. Mössbauer spectroscopy confirms the presence of octahedral and tetrahedral metal centers. A combination of DC susceptibility, ZFCM and Mossbauer studies reveals a magnetic phase transition to long-range order at $\sim 7 \mathrm{~K}$ in $\mathbf{1}$. Magnetic hysteresis is observed at low temperatures, with coersive fields of approximately 360 and $650 \mathrm{G}$ and remnant magnetizations of approximately 450 and $720 \mathrm{~cm}^{3} \mathrm{Gmol}^{-1}$ at 5 and $2.5 \mathrm{~K}$ respectively.
\end{abstract}




\section{Introduction}

Molecule-based magnets have been the subject of intense research over the last several decades. ${ }^{1-8}$ These materials undergo long-range ferromagnetic order below some critical temperature $\left(\mathrm{T}_{\mathrm{c}}\right)$, and materials within this class include complex metal cyanides, ${ }^{9-10}$ organometallic systems, ${ }^{10-11}$ and organic compounds ${ }^{12-14}$. A focus of our research, ${ }^{15-20}$ and others, ${ }^{21-23}$ includes coordination polymers of paramagnetic first-row transition metals incorporating bridging 1,3-diazolate ligands. In our own work we have documented examples of 1D, 2D and 3D extended lattice imidazolates which exhibit low temperature ferromagnetic ordering. Fe(1-Me-2-S-imid) ${ }_{2} \cdot 0.5 \mathrm{Cp}_{2} \mathrm{Fe}^{17,24}$ (where 1-Me-2-Simid = 1-methyl 2- thioimidazolate, and $\mathrm{Cp}=$ cyclopentadienyl), has a novel 1D extended chain structure incorporating alternating $\mathrm{FeN}_{4}$ and $\mathrm{FeS}_{4}$ tetrahedra. Examples with extended 3D lattice structures are $\mathrm{Fe}_{3}(\mathrm{imid})_{6}(\mathrm{Himid})_{2},{ }^{19} \mathrm{Fe}(4 \text {-abimid })_{2}{ }^{18}$ and $\mathrm{Fe}(2-$ meimid $)_{2} \cdot 0.13 \mathrm{Cp}_{2} \mathrm{Fe}^{20}($ where imid $=$ imidazolate, 4 -abimid $=4$-azabenzimidazolate, and 2-meimid $=2$-methylimidazolate). Finally, $\mathrm{Fe}_{2}(\mathrm{imid})_{4}($ bipy) 2 (where bipy $=2,2$ 'bipyridine) provides a $2 \mathrm{D}$ lattice structure example of these magnetic materials. ${ }^{15}$

Diazine ligands have been used frequently in coordination polymers for applications in sensing, ${ }^{25}$ optical and electronic materials, ${ }^{26}$ and molecular magnetism ${ }^{27-}$ 29. By incorporating the diazine ligand, 2,2'-bipyridine, into the iron(II) imidazolate structure we were able to cap some of the iron centers and in the process obtain the extended 2D lattice structure exhibited in $2 .{ }^{15} \quad$ Further studies on 2 show that the material magnetically orders with $\mathrm{T}_{\mathrm{c}} \sim 6.5 \mathrm{~K}$ and, in addition, undergoes two hysteretic structural and spin-state transitions starting at 151 and $133 \mathrm{~K}$ on cooling from room 
temperature. ${ }^{16}$ This remarkable behavior prompted the synthesis and study, reported here, of the 1,10-phenanthroline analogue, $\left[\mathrm{Fe}_{2}(\mathrm{imid})_{4}(\mathrm{phen})\right]_{\mathrm{x}}, \mathbf{1}$. In this study we fully characterize the extended structure of $\mathbf{1}$, and the magnetic ordering at low temperature.

\section{Experimental}

\subsection{Synthesis.}

Ferrocene $(0.30 \mathrm{~g}, 1.6 \mathrm{mmol})$, imidazole $(0.2 \mathrm{~g}, 2.9 \mathrm{mmol})$ and anhydrous 1,10phenanthroline $(0.15 \mathrm{~g}, 0.8 \mathrm{mmol})$ were placed in a Carius tube under a dry dinitrogen atmosphere, which was sealed under vacuum. The tube was heated at $130{ }^{\circ} \mathrm{C}$ for 4 days, then opened under a dry dinitrogen atmosphere. Following extraction of excess starting materials with acetonitrile and xylene solvents, the product was isolated as dark blue / brown moisture sensitive crystals suitable for X-ray crystallographic analysis. Yield $\sim 40$ \%. Analysis calculated for $\mathrm{C}_{24} \mathrm{H}_{20} \mathrm{Fe}_{2} \mathrm{~N}_{10}$ : C 51.5; H 3.6; N 25.0; found: C 51.8; H 3.7; N 25.3.

\subsection{Physical Measurements.}

Thermal gravimetric analysis (TGA) was done over the temperature range $35^{\circ} \mathrm{C}$ to $800{ }^{\circ} \mathrm{C}$ using a TA Instruments TA 2000 system with a TGA 51 unit. Powdered samples $(\sim 10 \mathrm{mg})$ were heated in a stream of dinitrogen at a rate of $10^{\circ} \mathrm{C} \mathrm{min}{ }^{-1}$.

DC Magnetic susceptibility measurements were made on powdered samples utilizing a Quantum Design (MPMS) SQUID magnetometer. The sample holder and details regarding the use of the equipment have been described elsewhere. ${ }^{30}$ Magnetic susceptibilities were corrected for the background signal of the sample holder and for 
diamagnetic susceptibilities of all atoms $\left(-142 \times 10^{-6} \mathrm{~cm}^{3} \mathrm{~mol}^{-1}\right)$. DC susceptibilities were measured from 2 to $300 \mathrm{~K}$ at 10000 and $500 \mathrm{G}$. Field-cooled, zero-field-cooled, and remnant DC magnetization measurements were made at an applied field of $50 \mathrm{G}$ and are reported over the temperature range $2-25 \mathrm{~K}$. Magnetic hysteresis was examined by cycling the applied field between +55000 and $-55000 \mathrm{G}$ at 2.5 and $5 \mathrm{~K}$. The data are reported on a per mole metal basis.

The Mössbauer spectra of powdered samples were determined using a conventional constant acceleration spectrometer operated in multi-channel scaling mode. The gamma ray source (Du Pont- Merck Co.) consisted of $51.5 \mathrm{mCi}$ of $\mathrm{Co}^{57}$ in a rhodium metal matrix that was maintained at ambient temperature. The spectrometer was calibrated using a 6-micron thick natural abundance iron foil. Isomer shifts are reported relative to the center of the magnetic hyperfine pattern of the latter foil taken as zero velocity. The line widths of the inner-most pair of $\Delta \mathrm{M}_{\mathbf{I}}= \pm 1$ transitions of the latter Zeeman pattern were reproducibly determined to be $0.214 \mathrm{~mm} / \mathrm{s}$. Sample temperature variation was achieved using a standard exchange gas liquid helium cryostat (Cryo Industries of America, Inc.) with temperature measurement and control based on silicon diode thermometry in conjunction with a 10 micro ampere excitation source (Lakeshore Cryotronics, Inc). Spectra were fit to unconstrained Lorentzians using the program Fityk. $^{31}$

X-ray Crystallographic studies were performed on a crystal of $\mathrm{C}_{24} \mathrm{H}_{20} \mathrm{Fe}_{2} \mathrm{~N}_{10}$ mounted on a glass fibre. All measurements were made on a Bruker X8 APEX diffractometer with graphite monochromated $\mathrm{Mo}-\mathrm{K} \alpha$ radiation and the structure was solved with SIR-97. ${ }^{32}$ All non-hydrogen atoms were refined anisotropically. All 
hydrogen atoms were included in calculated positions but not refined and crystal data information is included in Table 1. Diagrams were prepared using ORTEP-3. ${ }^{33}$

Table 1. Selected Crystalographic Data for $\mathbf{1}$.

\begin{tabular}{|c|c|}
\hline Chemical formula & $\mathrm{C}_{24} \mathrm{H}_{20} \mathrm{Fe}_{2} \mathrm{~N}_{10}$ \\
\hline Formula Mass & 560.20 \\
\hline Crystal system & Monoclinic \\
\hline$a / \AA$ & $9.2363(9)$ \\
\hline$b / \AA$ & $26.665(3)$ \\
\hline$c / \AA$ & $10.3514(12)$ \\
\hline$\alpha /{ }^{\circ}$ & 90.00 \\
\hline$\beta /^{\circ}$ & $107.041(4)$ \\
\hline$\gamma /{ }^{\circ}$ & 90.00 \\
\hline Unit cell volume $/ \AA^{3}$ & $2437.5(4)$ \\
\hline Temperature/K & $173(1)$ \\
\hline Space group & $P 21 / c$ \\
\hline No. of formula units per unit cell, $Z$ & 4 \\
\hline Radiation type & $\operatorname{MoK} \alpha$ \\
\hline Absorption coefficient, $\mu / \mathrm{mm}^{-1}$ & 1.225 \\
\hline No. of reflections measured & 31343 \\
\hline No. of independent reflections & 5805 \\
\hline $\boldsymbol{R}_{\text {int }}$ & 0.0590 \\
\hline Final $R_{1}$ values $(I>2 \sigma(I))$ & 0.0355 \\
\hline Final $w R\left(F^{2}\right)$ values $(I>2 \sigma(I))$ & 0.0715 \\
\hline Final $R_{1}$ values (all data) & 0.0600 \\
\hline Final $w R\left(F^{2}\right)$ values (all data) & 0.0767 \\
\hline Goodness of fit on $F^{2}$ & 1.052 \\
\hline
\end{tabular}

\section{Results and Discussion}

\subsection{Synthesis and Thermal Properties}

$\left[\mathrm{Fe}_{2}(\mathrm{imid})_{4}(\text { phen })\right]_{\mathrm{x}} \mathbf{1}$, was synthesized as brown / blue, moisture sensitive, crystals by the reaction of ferrocene with stoichiometric amounts of imidazole and phenanthroline in a Carius tube at $130{ }^{\circ} \mathrm{C}$. The synthetic strategy is similar to that 
employed earlier ${ }^{16}$ in the preparation of $\left[\mathrm{Fe}_{2}(\mathrm{imid})_{4}(\text { bipy })\right]_{\mathrm{x}} 2$ with the exception that, whereas an excess of the diazine in the reaction mixture gave pure samples of $\mathbf{2}$, pure samples of 1 could only be obtained when stoichiometric amounts of the diazine were used. The brown / blue crystals of $\mathbf{1}$ appear blue upon grinding to a fine powder. All characterizations were performed in a dry atmosphere and, with the exception of the Xray studies, were performed on powdered samples.

Compound $\mathbf{1}$ is thermally stable to about $295{ }^{\circ} \mathrm{C}$ and undergoes weight loss at higher temperatures in a two stage process. The first major weight loss $(\sim 40 \%)$ occurs between $295{ }^{\circ} \mathrm{C}$ and $400{ }^{\circ} \mathrm{C}$. The second weight loss ( 20\% of the initial weight) occurs between $450{ }^{\circ} \mathrm{C}$ and $500{ }^{\circ} \mathrm{C}$. The TGA does not show a plateau corresponding to the dissociation of phenanthroline ( $33 \%$ of the total weight). No further weight loss of any significance occurs up to $800{ }^{\circ} \mathrm{C}$. The residue left behind ( $\sim 30 \%$ of the original weight $)$ corresponds to the formation of $\mathrm{Fe}_{2} \mathrm{O}_{3}$.

\subsection{Structure}

The structure of $\mathbf{1}$ is that of a metal organic framework, or coordination polymer. The octahedral and tetrahedral Fe sites are shown in Figure 1. Crystallographic data are given in Table 1 and selected bond lengths and bond angles are given in Tables 2 and 3. 


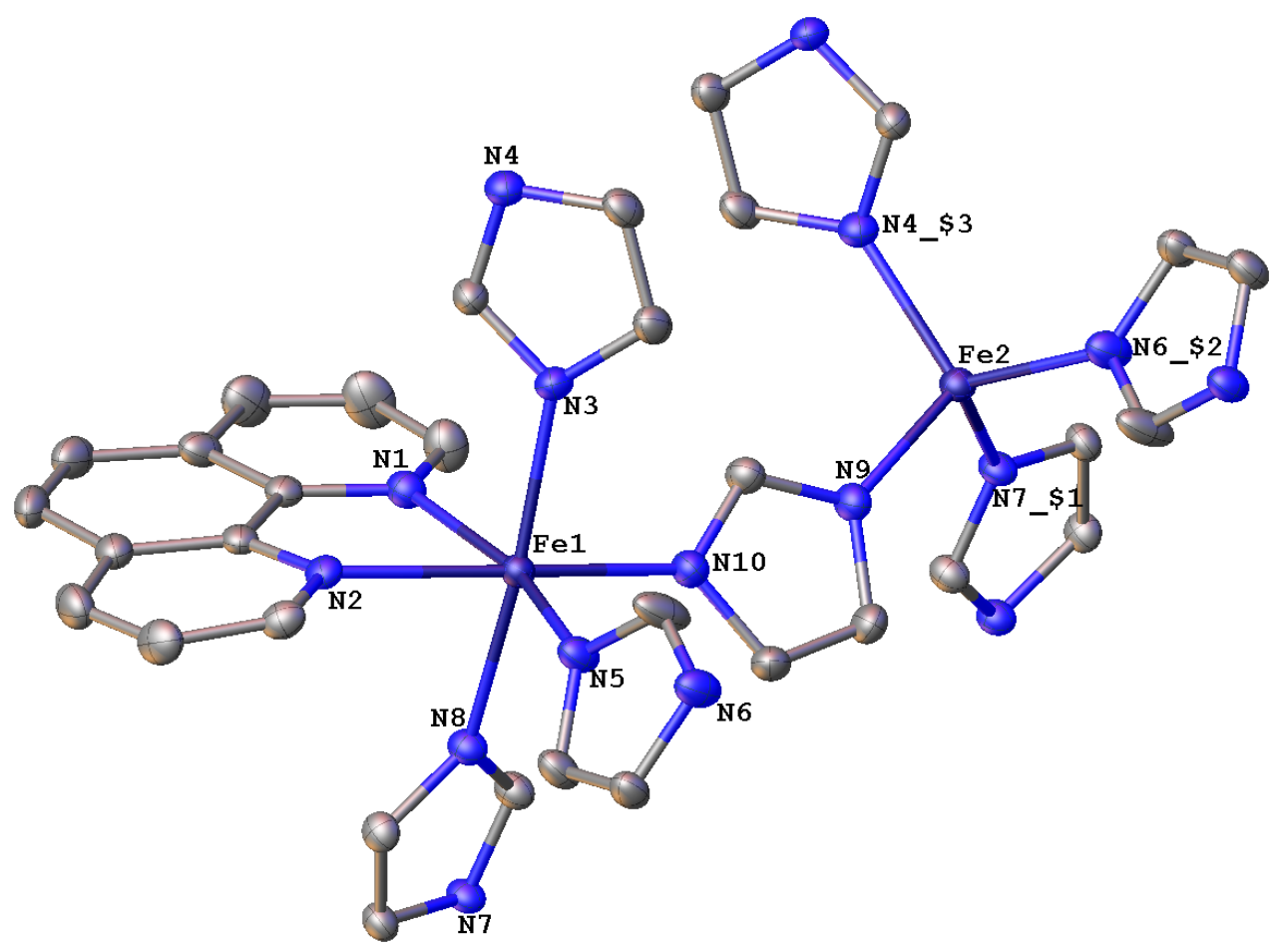

Figure 1: ORTEP plot of $\mathbf{1}$ ( $50 \%$ probability) excluding hydrogen atoms showing the octahedral and tetrahedral Fe sites in the extended structure. Atom colours: Fe, grey; $\mathrm{C}$, black; N, blue.

Table 2. Selected bond lengths $(\AA)$ for $\mathbf{1}$.

\begin{tabular}{ll}
\hline Fe1-N1 & $2.276(2)$ \\
Fe1-N2 & $2.248(2)$ \\
Fe1-N3 & $2.202(2)$ \\
Fe1-N5 & $2.124(2)$ \\
Fe1-N8 & $2.231(2)$ \\
Fe1-N10 & $2.137(2)$ \\
& \\
Fe2-N4 & $2.039(2)$ \\
Fe2-N6 & $2.036(2)$ \\
Fe2-N7 & $2.023(2)$ \\
Fe2-N9 & $2.041(2)$ \\
\hline
\end{tabular}


Table 3. Selected bond angles $\left(^{\circ}\right)$ for $\mathbf{1}$.

\begin{tabular}{ll}
\hline N1-Fe1-N2 & $73.68(7)$ \\
N1-Fe1-N3 & $87.04(7)$ \\
N1-Fe1-N5 & $165.51(7)$ \\
N1-Fe1-N8 & $83.88(7)$ \\
N1-Fe1-N10 & $97.78(7)$ \\
N2-Fe1-N3 & $94.82(7)$ \\
N2-Fe1-N5 & $91.89(7)$ \\
N2-Fe1-N8 & $83.71(7)$ \\
N2-Fe1-N10 & $170.76(7)$ \\
N3-Fe1-N5 & $95.60(7)$ \\
N3-Fe1-N8 & $170.85(7)$ \\
N3-Fe1-N10 & $88.12(7)$ \\
N5-Fe1-N8 & $93.47(7)$ \\
N5-Fe1-N10 & $96.55(7)$ \\
N8-Fe1-N10 & $92.00(7)$ \\
N4-Fe2-N6 & \\
N4-Fe2-N7 & $109.48(8)$ \\
N6-Fe2-N7 & $114.94(7)$ \\
N9-Fe2-N4 & $112.24(7)$ \\
N9-Fe2-N6 & $105.98(8)$ \\
N9-Fe2-N7 & $107.28(8)$ \\
\hline
\end{tabular}

The extended structure of 1 reveals both four- and six-coordinate iron centers. Each iron of one geometry is linked to four of the other type by single bridging imidazolates, forming double layer extended sheets, or bilayers. The 1,10-phenanthroline ligands coordinate as bidentate ligands at the pseudo-octahedral iron positions and occupy space between the bilayers. Two views of portions of the extended structure are shown in Figures 2 and 3. 


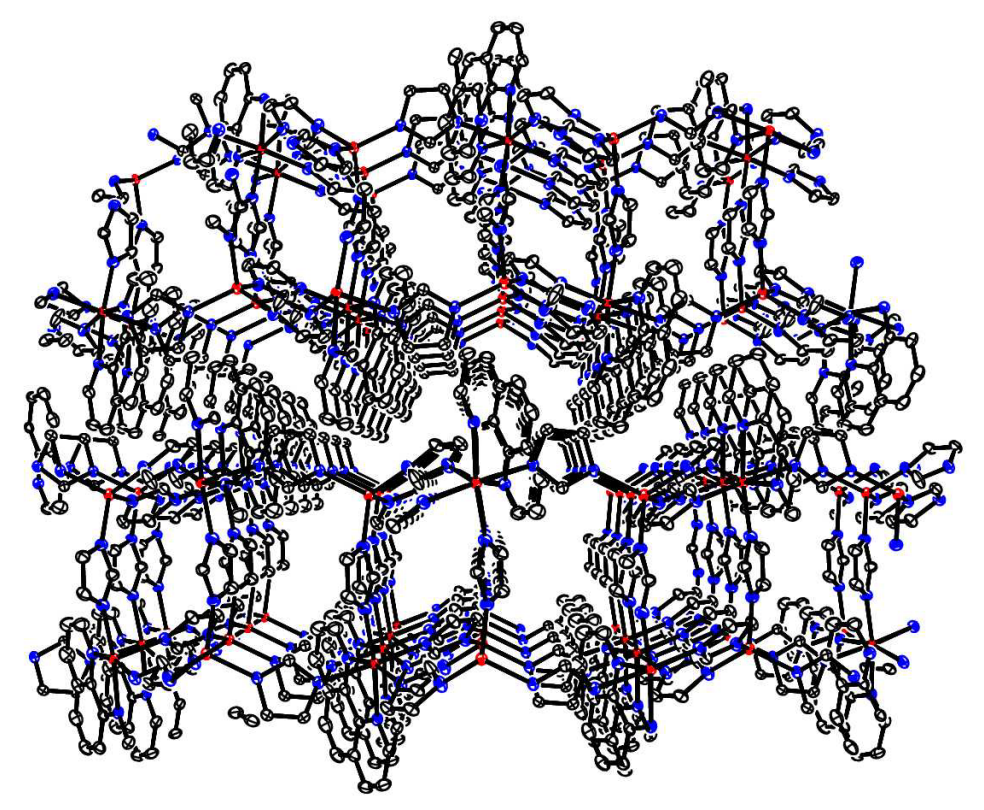

Figure 2: View parallel to two of the bilayer sheets in $\mathbf{1}$ showing phenanthroline positions.

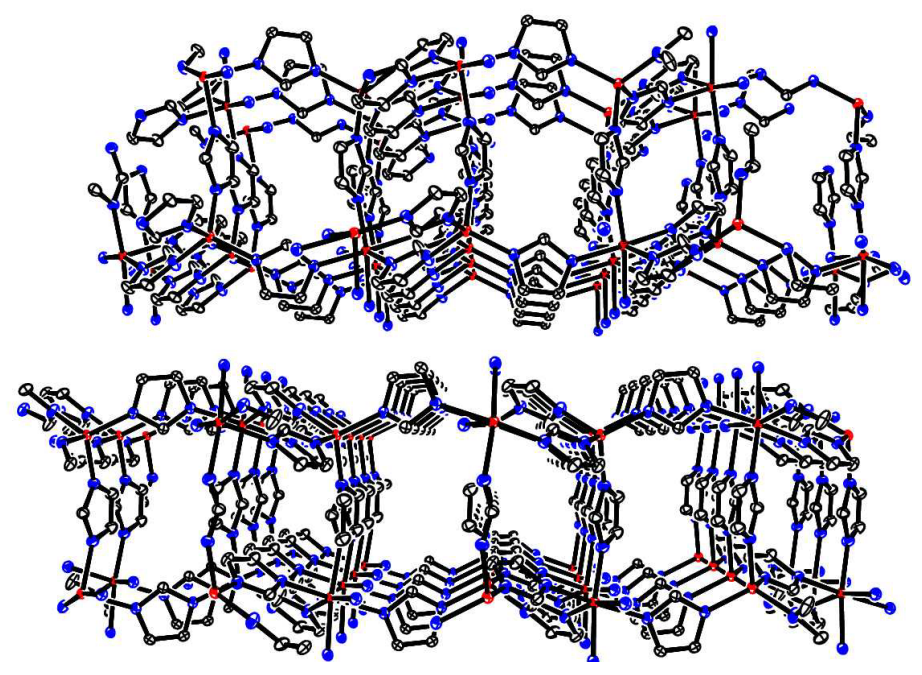

Figure 3: View parallel to two of the bilayer sheets in $\mathbf{1}$ with the phenanthrolines removed 
A simplified view of the overall structural motif is shown as a metal ion connectivity diagram in Figure 4. Three of the bilayers are shown in this picture. The top two bilayers have different orientations in the crystal. These two orientations alternate regularly throughout the lattice, the third bilayer in the figure having the same orientation as the first. In this figure octahedral irons are depicted as red and tetrahedral as green.

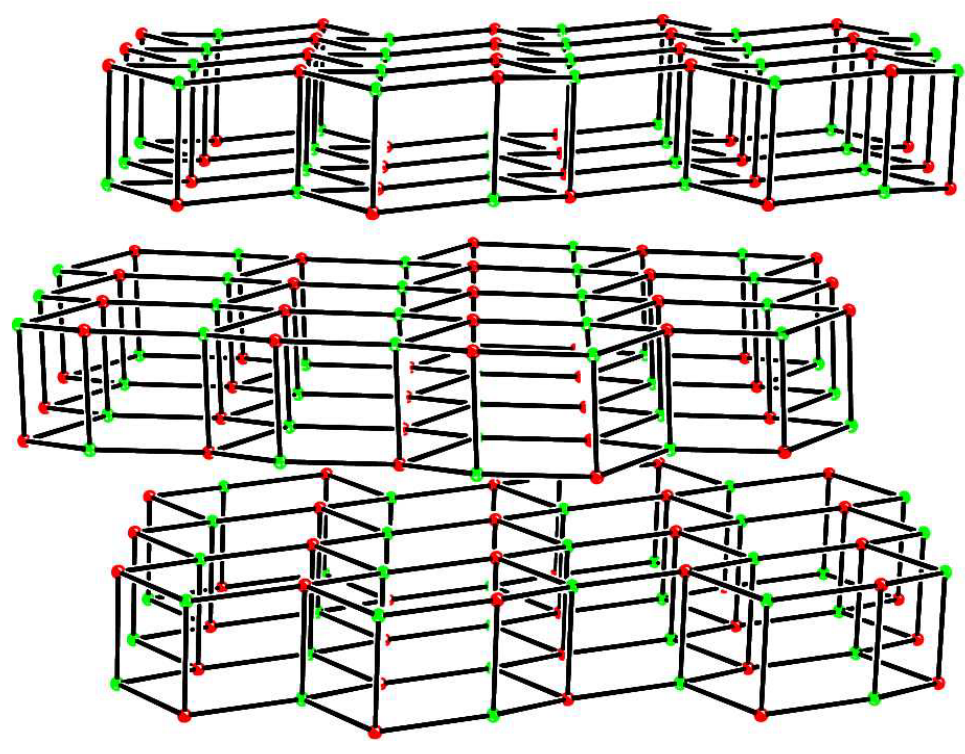

Figure 4: Iron ion connectivity diagram for a section of 1. Octahedral Fe (red) and tetrahedral Fe (green).

Overall, the structure of 1 closely matches that of the $\alpha$-form of the 2,2'bipyridine analogue, 2. ${ }^{16}$ The average $\mathrm{Fe}$ (oct) $-\mathrm{N}$ (Imidazolate) bond distance is 2.173(2) in $\mathbf{1}$ and 2.172(2) in $\mathbf{2}$, while the average Fe (tet) $-\mathrm{N}$ (imidazolate) distance is 2.035(2) in $\mathbf{1}$ and 2.028(2) in 2. The $\mathrm{Fe}-\mathrm{N}$ bond distances involving the different diazines are, as expected, different. The average value involving phen is 2.263(2) compared to 2.286(2) for bipy. As discussed in our earlier paper, ${ }^{16}$ these bond lengths are 
consistent with a high-spin configuration for the Fe (oct) sites as well as the Fe (tet) sites. The presence of two different bilayer orientations observed in $\mathbf{1}$ is not seen in $\mathbf{2}$, resulting in some significant differences in unit cell parameters. Notably, in $\mathbf{1}$, the $b$ axis is approximately twice as long and the unit cell volume twice as large as in $\mathbf{2}^{16}$

\subsection{Mossbauer spectroscopy}

The room temperature Mössbauer spectrum of 1 corresponds to two overlapping quadrupole doublets (Fig. 5A), and parameters (Table 4) consistent with pseudotetrahedral and pseudooctahedral high-spin iron(II). The intensity ratio of $c a .1$ (Table 4) is consistent with the X-ray structure.
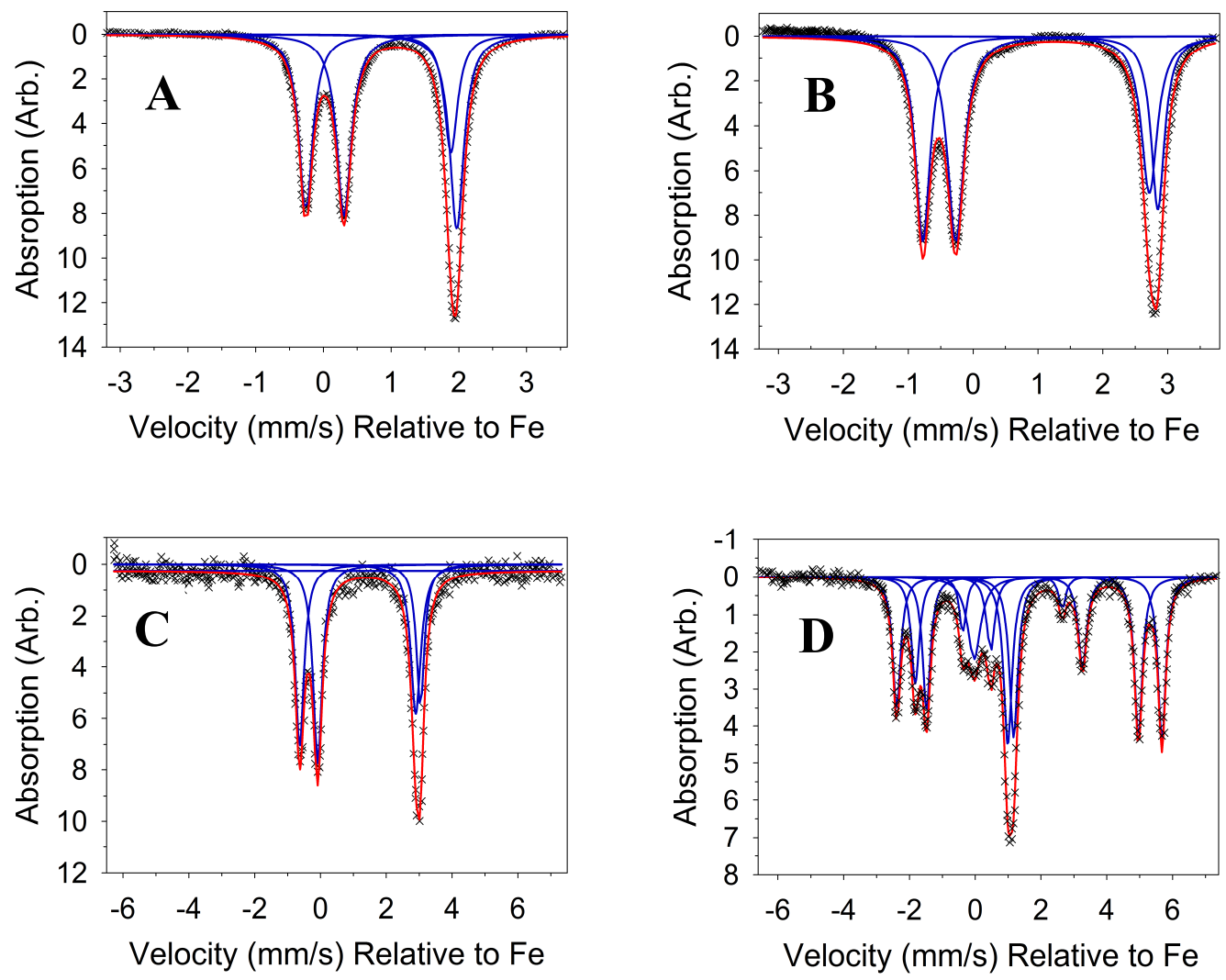

Figure 5: Mossbauer spectra of 1 at 293 K (A), 77.5 K (B), 12 K (C), and 4.2 K (D). 
The assignment of the $T_{d}$ (lines 1 and 4) and $O_{h}$ (lines 2 and 3) transition associations are consistent with isomer shifts reported for high-spin Fe(II) sites of similar ligation, ${ }^{15-16,34-35}$ however alternate transition associations $\left(1,3-T_{d}\right.$ and $\left.2,4-O_{h}\right)$ cannot be ruled out based on the present data. The $77.5 \mathrm{~K}$ (Figure 5B) and $12 \mathrm{~K}$ (Figure 5C) Mössbauer spectra are also consistent with two distinct high-spin Fe(II) sites in an approximate 1:1 ratio (Table 4).

Table 4: Mössbauer Spectroscopy Parameters

\begin{tabular}{lllll}
\hline$T, \mathrm{~K}$ & site & $\delta,^{\mathrm{a}} \mathrm{mm} / \mathrm{s}$ & $\Delta E, \mathrm{~mm} / \mathrm{s}$ & intensity, \% \\
\hline 293 & $O_{h}(2,3)^{b}$ & 1.09 & 1.58 & 45.2 \\
& $T_{d}(1,4)$ & 0.86 & 2.23 & 54.8 \\
77.5 & $O_{h}(2,3)$ & 1.22 & 2.99 & 52.7 \\
& $T_{d}(1,4)$ & 1.04 & 3.62 & 47.3 \\
$12^{c}$ & $O_{h}(2,3)$ & 1.41 & 2.99 & 54.7 \\
& $T_{d}(1,4)$ & 1.20 & 3.67 & 45.3 \\
4.2 & Sextet 1 & $-2.39,{ }^{d}-1.48,-0.037,0.99,2.64,4.96$ & 53.7 \\
& Sextet 2 & $-1.82,-0.38,0.47,1.17,3.26,5.67$ & 46.3 \\
\hline
\end{tabular}

${ }^{a}$ Relative to natural Fe foil at $293 \mathrm{~K}$. ${ }^{b}$ The integers refer to the transitions in Figure XX reading left to right. ${ }^{c}$ Baseline correction used. ${ }^{d}$ Values correspond to the individual peak centers.

As expected, the isomer shifts increase upon cooling from $293 \mathrm{~K}$ to $77.5 \mathrm{~K}$ and 12 $\mathrm{K}$ due to the second-order Doppler effect. In addition, the quadrupolar splitting $(\Delta E)$ increases as the temperature is lowered. The temperature dependence of the quadrupolar splitting can be attributed to thermal averaging of the electron configuration at higher temperatures and/or changes in the orientation of the electric field gradient (EFG) tensor. $^{36-37}$ The three spectra (Figures 5A, 5B and 5C) are consistent with the absence of any structural phase transitions from ambient temperature down to $12 \mathrm{~K}$. This is in sharp contrast to the situation observed for the bipy analogue, 2, where spectral changes 
consistent with transitions from $\alpha$ to $\beta$ to $\gamma$ phases were clearly observed over this temperature range. ${ }^{16}$

Significant changes in the Mössbauer spectrum are visible at $4.2 \mathrm{~K}$, and the data suggest the presence of two sextets (total of 12 lines) indicating magnetic ordering of the complex. Sample ordering is in line with the magnetic data, discussed below, showing a phase transition to long range order below $c a .7 .5 \mathrm{~K}$. We have further evaluated the hyperfine transitions to determine the effective hyperfine field $\left(H_{n}\right)$ at the high spin $\mathrm{Fe}(\mathrm{II})$

sites. ${ }^{38-39}$ We can estimate $H_{n}$ from the overall spectral splitting (in this case $\Delta_{1-6}$ is 7.35 and $7.49 \mathrm{~mm} / \mathrm{s}$ for the two sextets) affording a value of $\sim 23$ tesla for both $\mathrm{Fe}$ (II) sites, which is in line for reported high spin Fe(II) hyperfine fields. ${ }^{40-41}$ Overall, the Mössbauer data support the assignment of two distinct pseudotetrahedral and pseudooctahedral high spin $\mathrm{Fe}(\mathrm{II})$ centers above the ordering temperature of ca. $7.5 \mathrm{~K}$, with two six line patterns consistent with long range order in the material at $4.2 \mathrm{~K}$.

\subsection{Magnetic properties}

DC magnetic susceptibility studies on a powdered sample of $\mathbf{1}$ at an applied field of $10,000 \mathrm{G}$ reveal antiferromagnetic behavior, the moment decreasing on cooling from $300 \mathrm{~K}$ to $2 \mathrm{~K}$ (Figure 6). On close examination (Figure 6 insert), the moment shows an anomaly at about $10 \mathrm{~K}$. 


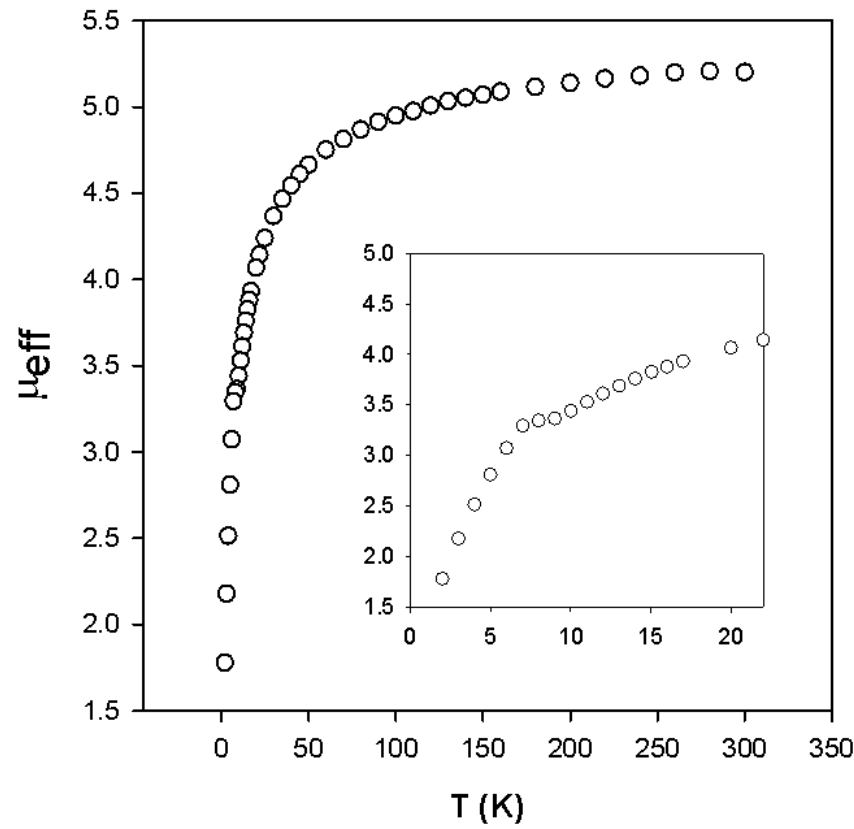

Figure 6: $\mu_{\text {eff }}$ versus temperature plots at $10000 \mathrm{G}$ for $\mathbf{1}$.

Measurements at an applied field of 500G (Figure 7 and insert) reveal that at this reduced applied field the moment decreases on cooling to about $9 \mathrm{~K}$ below which it increases abruptly indicative of ferromagnetic ordering. Both 1 and the 2,2'-bipyridine analogue, $2,{ }^{15}$ exhibit antiferromagnetic exchange at high temperatures coupled with a net moment ground state, classifying them as weak ferromagnets. The onset of a transition to a magnetically ordered state at low temperatures was confirmed for $\mathbf{1}$ by zero field cooled magnetism (ZFCM), field cooled magnetism (FCM) and remnant magnetism (REM) studies in a DC field of 50G. All three measurements (Figure 8) reveal abrupt transitions at $\sim 7 \mathrm{~K}$, thus providing a good measure of $\mathrm{T}_{\mathrm{c}}$. Magnetic hysteresis was observed for $\mathbf{1}$ at temperatures below Tc. Cycling the applied magnetic field between + 55000 and $-55000 \mathrm{G}$ at $5 \mathrm{~K}$ generates a hysteresis loop with a coersive field of $\sim 360 \mathrm{G}$ 
and a remnant magnetization of $\sim 450 \mathrm{~cm}^{3} \mathrm{Gmol}^{-1}$. The hysteresis loop obtained at $2.5 \mathrm{~K}$ is shown in Figure 9 (and insert). From this loop and a coercive field of $\sim 650 \mathrm{G}$ and remnant magnetization of $\sim 720 \mathrm{~cm}^{3} \mathrm{Gmol}^{-1}$ are obtained.

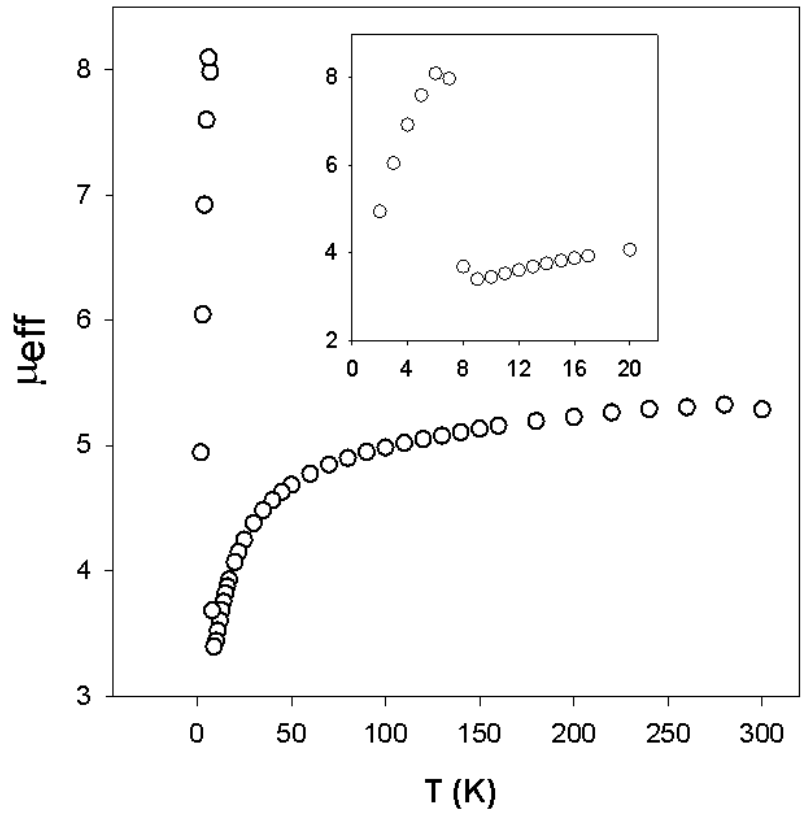

Figure 7: $\mu_{\text {eff }}$ versus temperature plots at $500 \mathrm{G}$ for $\mathbf{1}$.

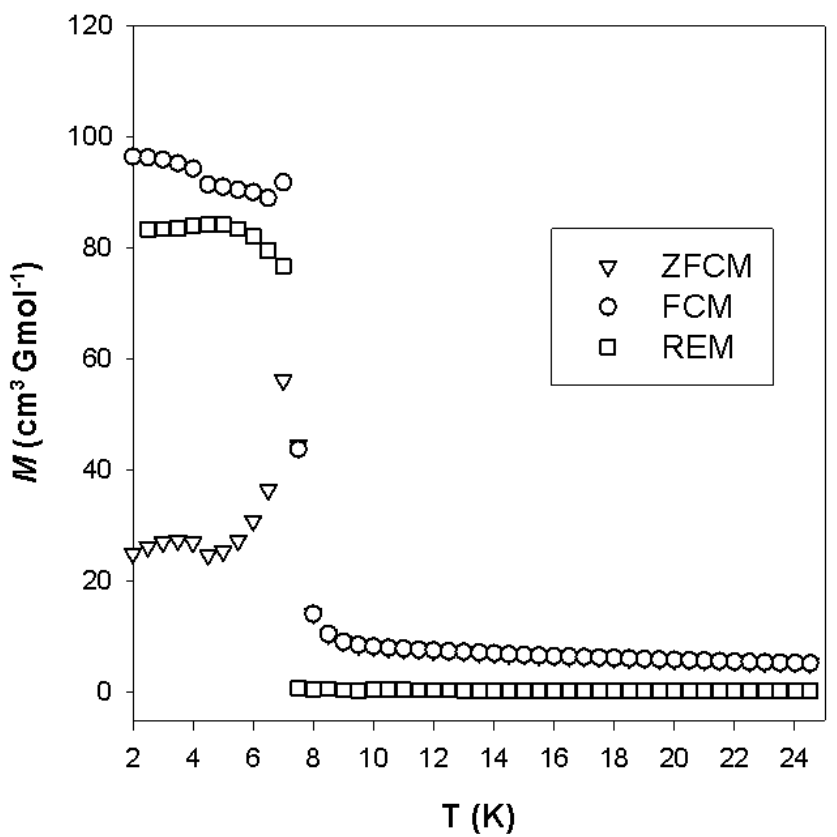

Figure 8: ZFCM, FCM and REM plots at 50G for $\mathbf{1}$. 


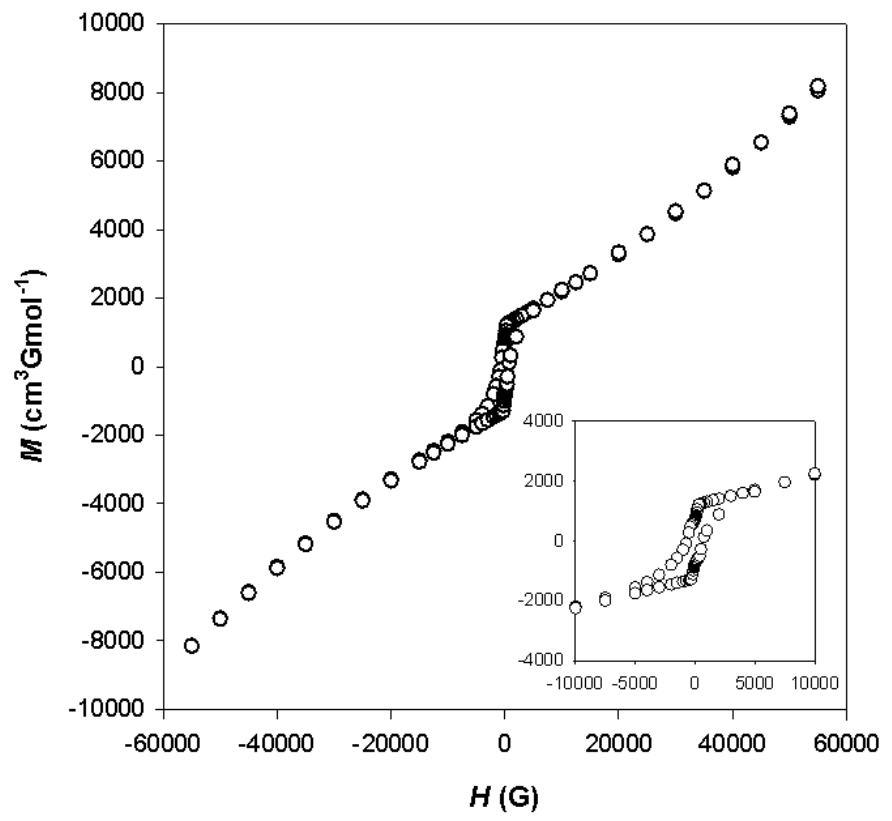

Figure 9: Magnetic hysteresis plot at 2.5K for 1.

Two possible causes of the weak ferromagnet properties of $\mathbf{1}$ are (i) spin canting and (ii) ferrimagnetism. In both, the primary magnetic interaction is of an antiferromagnetic nature. In (i) antiparallel alignment of neighboring spins is not perfect due to a canting of the spins. ${ }^{42}$ In (ii) antiferromagnetically interacting magnetic dipoles of different sizes alternate regularly throughout the lattice. ${ }^{42}$ In both cases, a residual spin remains on the lattice as the temperature is lowered and long-range ferromagnetic ordering of these spins occurs below the critical temperature, $\mathrm{T}_{\mathrm{c}}$. Either or both of these mechanisms could contribute to the magnetic behavior of 1. Interestingly, while examples of ferrimagnetism usually involve the regular alternation of different metals, ${ }^{42}$ it is possible in the case of a single metal where different oxidation states or different ligand chromophores lead to different $g$ values and therefore different size magnetic 
dipoles. The latter situation would apply to $\mathbf{1}$ which has a structure supporting magnetic interactions between octahedral and tetrahedral iron(II) centers. The possibility of either one or both of the mechanisms (i) and (ii) being applicable to a given weak ferromagnet has been discussed previously for the compounds $\mathbf{2}^{15}$ and polybis(1-methyl-2thioimidazolate)iron(II) ${ }^{17}$

\section{Summary and Conclusion}

Single crystal X-ray diffraction studies on the title compound, 1, revealed an extended MOF structure consisting of double layer sheets of alternating tetrahedral and octahedral iron(II) centers bridged by imidazolate ligands, with the octahedral sites additionally coordinated by chelating 1,10-phenanthroline molecules. The presence of equal numbers of octahedral and tetrahedral iron(II) sites was confirmed via Mossbauer spectroscopy. The extended structure is very similar to that of the previously studied 2,2'bipyridine analogue, $2{ }^{15-16}$ Only modest differences are seen in the $\mathrm{Fe}-\mathrm{N}$ (imidazolate) bond distances and chromophore geometries in the two compounds. In spite of these equivalences no structural phase transitions, of the kind seen for $\mathbf{2}$, are observed on cooling 1 from $300 \mathrm{~K}$ to $2 \mathrm{~K}$. X-ray diffraction studies on the three structural phases of 2 revealed modest differences in chromophore geometries in the three phases as well as differences in molecular orientations of the ligands about the octahedral centers, which

explained facile conversion of one phase to another while maintaining crystal integrity. ${ }^{16}$

Since 1 does not exhibit these changes in molecular structure on cooling, this suggests that the size and flexibility of the diazine ligand is key to the phenomenon which occurs 
with the relatively flexible $2-2^{\prime}$-dipyridine but not with the larger and more rigid 1,10phenanthroline.

Variable temperature DC magnetization and Mossbauer spectroscopy studies of 1 from $300 \mathrm{~K}$ to $2 \mathrm{~K}$ gave no evidence for structural phase transitions over the temperature range studied but both confirmed a transition to a ferromagnetically ordered state at low temperatures. ZFCM, FCM and REM studies determined a $T_{c}$ value of $\sim 7 \mathrm{~K}$. Mossbauer measurements were also consistent with magnetic ordering below $12 \mathrm{~K}$ with an effective hyperfine field value of $\sim 23$ tesla for both Fe sites at $4.2 \mathrm{~K}$. Magnetic hysteresis measurements at $2.5 \mathrm{~K}$ gave a coersive field of $\sim 650 \mathrm{G}$ and a remnant magnetization of $\sim 720 \mathrm{~cm}^{3} \mathrm{Gmol}^{-1}$. The overall similarities in the extended structures of $\mathbf{1}$ and the 2,2'-bipyridine analogue, $\mathbf{2}$, probably account for the fact that both exhibit the properties of a weak ferromagnet with similar $T_{c}$ values. Either spin canting or ferrimagnetism, or some combination of the two, probably generate the observed weak ferromagnet behavior. The two compounds differ somewhat in their hysteresis properties. Measurements on 1 at $5 \mathrm{~K}$ gave a coersive field of $\sim 360 \mathrm{G}$ and a remnant magnetization of $\sim 450 \mathrm{~cm}^{3} \mathrm{Gmol}^{-1}$ while, at $4.8 \mathrm{~K}, 2$ showed values of $\sim 15 \mathrm{G}$ and $\sim 200 \mathrm{~cm}^{3} \mathrm{Gmol}^{-1}$ respectively. ${ }^{15}$

\section{Acknowledgements}

A.S. and R.C.T. thank the Natural Sciences and Engineering Research Council of Canada for financial support. W.M.R. is pleased to acknowledge the US NSF for funding. 


\section{References:}

(1) Dechambenoit, P.; Long, J. R. Chem. Soc. Rev. 2011, 40, 3249.

(2) Hardy, J. G. Chem. Soc. Rev. 2013, 42, 7881.

(3) Leong, W. L.; Vittal, J. J. Chem. Rev. 2011, 111, 688.

(4) Murray, K. S. Aust. J. Chem. 2009, 62, 1081.

(5) Kahn, O. Acc. Chem. Res. 2000, 33, 647.

(6) Miller, J. S. Chem. Soc. Rev. 2011, 40, 3266.

(7) Bagai, R.; Christou, G. Chem. Soc. Rev. 2009, 38, 1011.

(8) Gatteschi, D. Adv. Mat. 1994, 6, 635.

(9) Miller, J. S.; Manson, J. L. Acc. Chem. Res. 2001, 34, 563.

(10) Ferlay, S.; Mallah, T.; Ouahes, R.; Veillet, P.; Verdaguer, M. Nature 1995, 378, 701.

(11) Miller, J. S. Inorg. Chem. 2000, 39, 4392.

(12) Banister, A. J.; Bricklebank, N.; Lavender, I.; Rawson, J. M.; Gregory, C. I.; Tanner, B. K.; Clegg, W.; Elsegood, M. R. J.; Palacio, F. Angew. Chem. Int. Ed. 1996, 35, 2533.

(13) Naber, W. J. M.; Faez, S.; van der Wiel, W. G. J. Phys. D-Appl. Phys. 2007, 40, R205.

(14) Miller, J. S. Chem. Eur. J. 2015, 21, 4506.

(15) Patrick, B. O.; Reiff, W. M.; Sanchez, V.; Storr, A.; Thompson, R. C. Polyhedron 2001, 20, 1577.

(16) Patrick, B. O.; Reiff, W. M.; Sanchez, V.; Storr, A.; Thompson, R. C. Inorg. Chem. 2004, 43, 2330.

(17) Rettig, S. J.; Sanchez, V.; Storr, A.; Thompson, R. C.; Trotter, J. Inorg. Chem. 1999, 38,5920 .

(18) Rettig, S. J.; Sanchez, V.; Storr, A.; Thompson, R. C.; Trotter, J. J. Chem. Soc. Dalton Trans. 2000, 3931.

(19) Rettig, S. J.; Storr, A.; Summers, D. A.; Thompson, R. C.; Trotter, J. J. Am. Chem. Soc. 1997, 119, 8675. 
(20) Rettig, S. J.; Storr, A.; Summers, D. A.; Thompson, R. C.; Trotter, J. Can. J. Chem. 1999, 77, 425 .

(21) Patel, R.; Weller, M. T.; Price, D. J. Dalton Trans. 2007, 4034.

(22) Herchel, R.; Sindelar, Z.; Travnicek, Z.; Zboril, R.; Vanco, J. Dalton Trans. 2009, 9870 .

(23) Zhu, X.; Wang, X. Y.; Li, B. L.; Wang, J.; Gao, S. Polyhedron 2012, 31, 77.

(24) Reiff, W. M.; Sanchez, V.; Thompson, R. C.; Storr, A. Polyhedron 2001, 20, 1243.

(25) Katz, M. J.; Sakai, K.; Leznoff, D. B. Chem. Soc. Rev. 2008, 37, 1884.

(26) Achelle, S.; Ple, N.; Turck, A. RSC Advances 2011, 1, 364.

(27) Thompson, L. K.; Dawe, L. N. Coord. Chem. Rev. 2015, 289, 13.

(28) Bhatt, P.; Thakur, N.; Meena, S. S.; Mukadam, M. D.; Yusuf, S. M. J. Mat. Chem. C 2013, $1,6637$.

(29) Bhatt, P.; Thakur, N.; Mukadam, M. D.; Meena, S. S.; Yusuf, S. M. J. Phys. Chem. C 2014, 118, 1864.

(30) Ehlert, M. K.; Rettig, S. J.; Storr, A.; Thompson, R. C.; Trotter, J. Can. J. Chem. 1989, 67, 1970.

(31) Wojdyr, M. J. Appl. Crystallogr. 2010, 43, 1126.

(32) SIR97; Altomare, A.; Burla, M. C.; Camalli, M.; Cascarano, G. L.; Giacovazzo, C.; Guagliardi, A.; Moliterni, A. G. G.; Polidori, G.; Spagna, R. J. Appl. Crystallogr. 1999, $32,115$.

(33) Farrugia, L. J. J. Appl. Crystallogr. 2012, 45, 849.

(34) Long, G. J.; Grandjean, F.; Trofimenko, S. Inorg. Chem. 1993, 32, 1055.

(35) Reger, D. L.; Little, C. A.; Rheingold, A. L.; Sommer, R.; Long, G. J. Inorg. Chim. Acta 2001, 316, 65.

(36) Suzuki, T.; Okubo, N. Z. Naturforsch. A 2002, 57, 627.

(37) Vanalboom, A.; Degrave, E.; Vandenberghe, R. E. Hyperfine Interactions 1994, 91, 703.

(38) Van Dongen Torman, J.; Jagannathan, R.; Trooster, J. M. Hyperfine Interact. 1975, $1,135$. 
(39) Walton, E. G.; Brown, D. B.; Wong, H.; Reiff, W. M. Inorg. Chem. 1977, 16, 2425.

(40) Cooke, R. In Mossbauer effect methodology; Gruverman, I. J., Ed.; Springer: New York, 2013; Vol. 4, p 113.

(41) Ono, K.; Ito, A. J. Phys. Soc. Jpn. 1964, 19, 899.

(42) Kahn, O. Molecular Magnetism; VCH: New York, 1993.

\section{TOC Entry:}

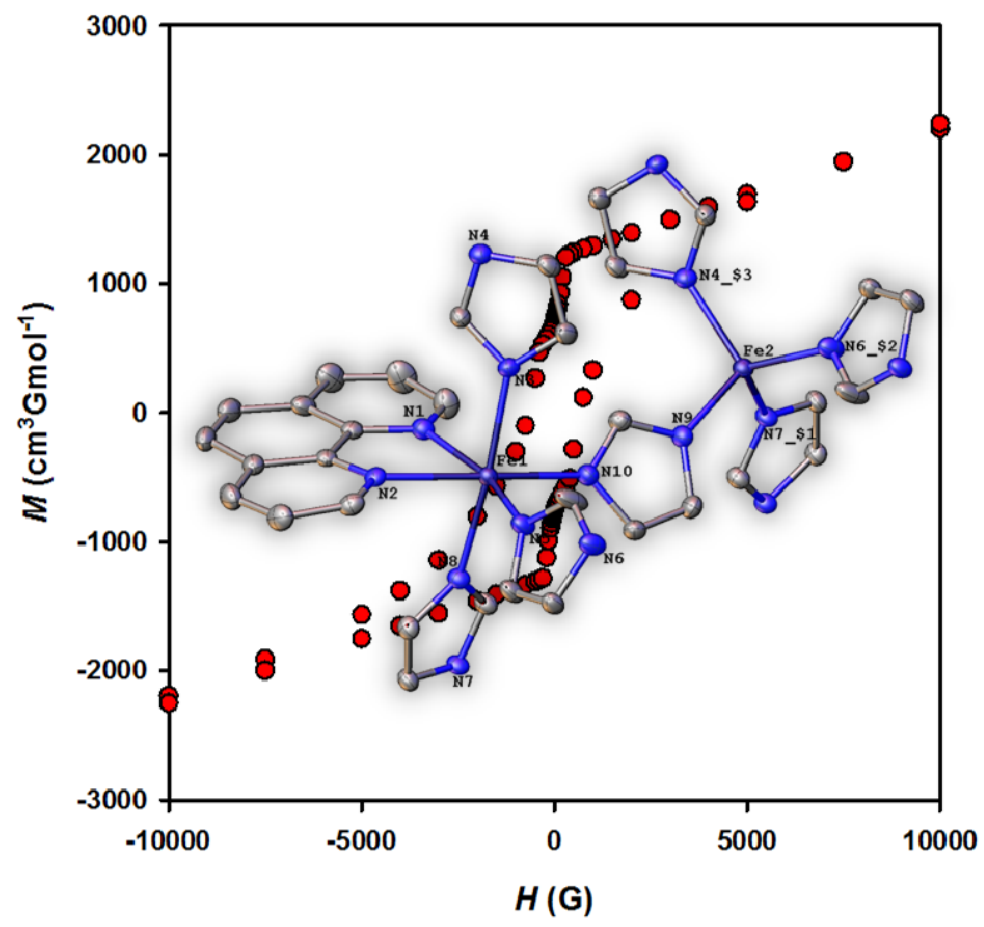

